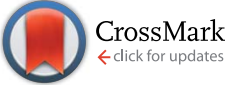

Cite this: RSC AdV., 2017, 7, 17741

Received 10th February 2017

Accepted 13th March 2017

DOI: $10.1039 / \mathrm{c} 7 \mathrm{ra01722g}$

rsc.li/rsc-advances

\section{Dichroic nonlinear absorption response of silver nanoprism arrays}

\author{
Tiziana Cesca, ${ }^{\text {aa }}$ Emma Vianey García-Ramírez, ${ }^{b}$ Hectór Sánchez-Esquivel, ${ }^{c}$ \\ Niccolò Michieli, ${ }^{a}$ Boris Kalinic, ${ }^{a}$ Juan Manuel Gómez-Cervantes, ${ }^{b}$ Raul Rangel-Rojo, ${ }^{c}$ \\ Jorge Alejandro Reyes-Esqueda ${ }^{\mathrm{b}}$ and Giovanni Mattei ${ }^{\mathrm{a}}$
}

\begin{abstract}
The properties of dichroism in the nonlinear absorption response of silver nanoprism arrays are experimentally investigated by the z-scan technique. Open aperture z-scan measurements are performed with a picosecond laser at a low repetition rate to excite the third-order electronic component of the optical nonlinearity for different linear polarization directions of the incident beam. A strong saturable absorber behavior is observed for all the linear polarization orientations and a continuous, sinusoidal modulation of the nonlinear absorption coefficient (up to about $30 \%$ of its absolute value) is demonstrated as a function of the polarization angle. The dichroic nonlinear absorption of the samples is explained by taking into account the polarization-dependent local electricfield distribution in the nanoprism arrays, as demonstrated by finite elements method electrodynamic simulations. These findings make silver nanoprism arrays an ideal platform for the development of nanophotonic devices with fast, strong and tunable nonlinear optical responses.
\end{abstract}

Recent years have seen a tremendous burst of research activity towards the development of novel nonlinear optical (NLO) materials for advanced applications in photonics and optoelectronics. ${ }^{1-5}$ From this point of view, plasmonic nanomaterials are of great interest for their strong and ultra-fast nonlinear response. ${ }^{6}$ By coupling light to surface plasmons, an intense enhancement and the confinement of the local electromagnetic field at the plasmonic nanostructures can be obtained, ${ }^{7}$ which is of paramount importance to develop metamaterials with properly tuned and enhanced optical nonlinearity ${ }^{8-13}$ In recent years a variety of different plasmonic nanosystems, both with random distribution and as ordered arrays, have been developed and characterized for NLO applications. ${ }^{14-25}$ Among them, 2D ordered nanoprism arrays (NPAs) represent a very interesting configuration. ${ }^{26,27}$ The confinement of the electromagnetic field at the nanoprisms' sharp tips gives rise to the formation of intense hot-spots (further amplified by near-field interactions among the nanoprisms in the array) that significantly boost the nonlinear optical response of these nanomaterials. ${ }^{18,26,28}$

\footnotetext{
${ }^{a}$ Department of Physics and Astronomy, University of Padova, via Marzolo 8, I-35131 Padova, Italy. E-mail: tiziana.cesca@unipd.it

${ }^{b}$ Instituto de Física, Universidad National Autónoma de México, Circuito de la Investigación Cientifica, Ciudad Universitaria, Delegación Coyoacán, C.P. 4510, Ciudad de México, México

'División de Física Aplicada, Centro de Investigación Científica y de Educación Superior de Ensenada, Carretera Ensenada-Tijuana No. 3918, Zona Playitas, C.P. 22860, Ensenada, México
}

Besides a strong and ultra-fast nonlinear optical response, a very important requirement for NLO applications is the capability to control and modulate the material nonlinearity in a desired way. For plasmonic nanosystems, an effective approach to obtain such a control is by properly engineering the structural and compositional parameters of the nanomaterials. ${ }^{25,26,29}$ As an example, regarding the nanoprism array configuration, we recently demonstrated that the nonlinear absorption properties of bimetallic $\mathrm{Au}-\mathrm{Ag}$ NPAs can be varied by properly selecting the bimetallic composition of the nanoprisms, without changing any other structural parameter of the nanoarray. ${ }^{26}$ On the other hand, the capability to modify the nonlinear response of nanomaterials in a reversible way by switching on some external parameters would be of great interest for NLO applications. To this aim, in the present work we studied the possibility of using the linear polarization of a laser beam to get the desired external control of the material nonlinearity. To do this, we investigated the effects of dichroism in the nonlinear absorption response of silver NPAs synthesized via nanosphere lithography. ${ }^{30}$ Nonlinear optical characterization was performed using z-scan measurements. ${ }^{31} \mathrm{~A}$ picosecond laser source at a low repetition rate was used to excite the fast, electronic, third-order component of the nonlinear response of the NPAs, inducing minimal thermal load to the samples. Strong saturable absorber (SA) behavior was observed for all the polarization orientations of the laser beam explored. Moreover, it was demonstrated that a continuous modulation of the nonlinear absorption coefficient can be obtained by rotating the polarization, leaving at the same time the linear absorption 
properties of the nanomaterial globally unaffected. The linear polarization of an external laser beam therefore represents a useful degree of freedom to get the external fine control of the material nonlinear absorption properties. These findings, combined with the cost-effectiveness and high throughput of the nanosphere lithography technique used for the synthesis of the samples, make silver nanoprism arrays a very interesting and highly promising platform for the development of alloptical nanophotonic devices with fast, strong and tunable nonlinear optical responses.

\section{Experimental section}

\subsection{Synthesis of nanoprism arrays}

Silver nanoprism arrays (NPAs) were produced using the nanosphere lithography (NSL) technique. ${ }^{\mathbf{2 6 , 3 0 , 3 2}}$ The main steps of fabrication are schematically illustrated in Fig. 1a. First, a colloidal monolayer of polystyrene (PS) nanospheres was formed, by self-assembling, on top of a silica glass substrate previously cleaned in a "piranha" solution $\left(\mathrm{H}_{2} \mathrm{SO}_{4}: \mathrm{H}_{2} \mathrm{O}_{2}, 3: 1\right)$. Commercial PS nanospheres (Microparticles $\mathrm{GmbH}$ ) with a nominal diameter of $248 \mathrm{~nm}$ were used. As the second step, silver was thermally evaporated in an orthogonal configuration on the PS monolayer. The thickness of the evaporated layer was controlled with a calibrated quartz microbalance. The PS nanospheres were then mechanically removed by stripping with an adhesive tape. After this step, an ordered array of triangular Ag nanoprisms, the same as the one shown in Fig. 1b, is left on the surface of the silica substrates. Finally, to protect the nanoprism arrays from possible physical damage and $\mathrm{Ag}$ oxidation (which would alter the optical properties of the samples), a layer of silica with thickness of about $160 \mathrm{~nm}$ was deposited on the samples by magnetron sputtering.

\subsection{Structural and linear optical characterizations}

Morphological characterizations of the NPAs were performed using scanning electron microscopy (SEM). The measurements were made with a Zeiss Sigma field-emission scanning electron microscope (FE-SEM) working in the 0.2-30 kV range. An energy-dispersive spectrometer (EDS) is also mounted in the microscope for compositional analysis. Atomic force microscopy (AFM) measurements were performed to measure the height of the nanoprisms. A NT-MDT Solver PRO-M AFM microscope with a $100 \times 100 \mu \mathrm{m}$ scanner was used, operated in the semi-contact mode.

Optical absorbance spectra in the 300-900 wavelength range were collected using a dual beam UV-Vis spectrophotometer (JASCO V670) with unpolarized light. Absorbance measurements as a function of the incident polarization orientation were also performed using an Ocean Optics USB2000 spectrometer coupled with optical fibers to a deuterium-halogen dual lamp.

\subsection{Nonlinear absorption measurements}

The third-order nonlinear absorption properties of the synthesized nanoprism arrays were investigated using open aperture (OA) z-scan measurements. ${ }^{31}$ The measurements were performed using the second harmonic $(\lambda=532 \mathrm{~nm})$ of a picosecond pulsed mode-locked Nd:YAG laser (pulse duration of 26 (a)

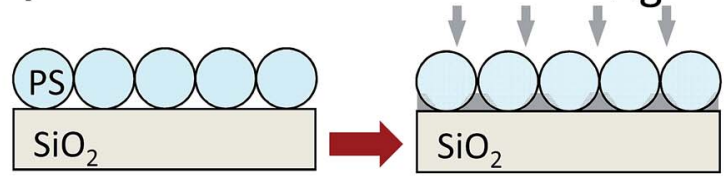

1. Nanosphere self-assembly

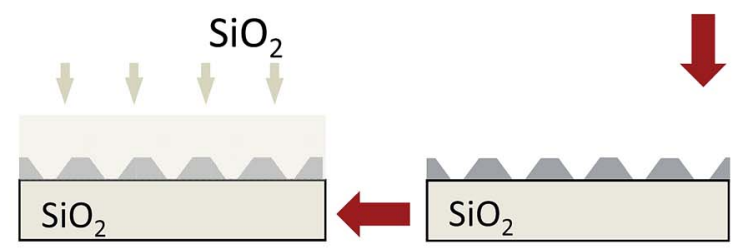

4. $\mathrm{SiO}_{2}$ sputtering deposition

3. Nanosphere removal
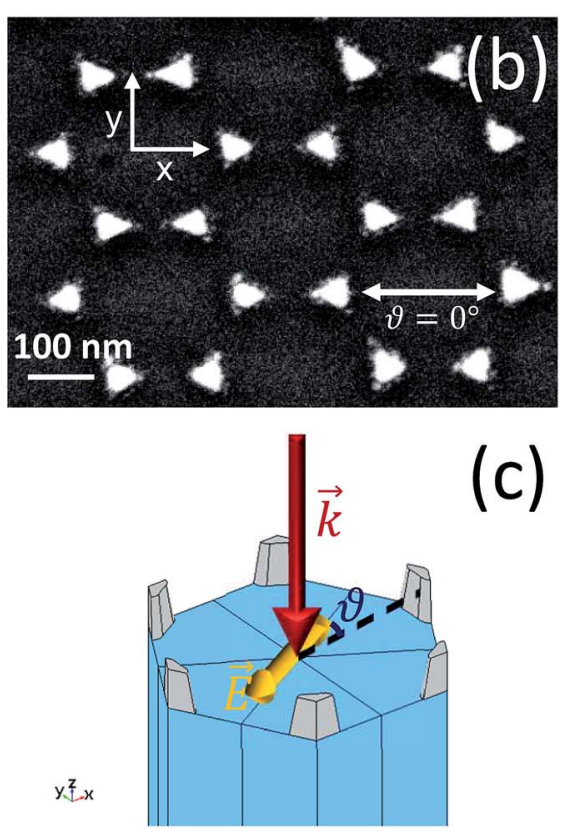

Fig. 1 (a) Schematic illustration of the different steps for the synthesis of silver nanoprism arrays: (1) colloidal mask formation via the selfassembly of PS nanospheres on a silica substrate; (2) Ag thermal evaporation on the PS nanosphere mask; (3) nanosphere removal; (4) deposition by magnetron sputtering of a protecting layer of silica. (b) FE-SEM image of a Ag nanoprism array. (c) Sketch of the 3D geometry of the FEM model. The incident light beam is orthogonal to the sample surface, and the electric field is linearly polarized forming an angle $\theta$ with respect to the $\hat{x}$ direction. 
ps, repetition rate $10 \mathrm{~Hz}$ ). A $400 \mathrm{~mm}$ converging lens was used to focus the laser beam in the z-scan set-up. The beam waist $w_{0}$ at the lens focal point was measured using the knife-edge method $^{33}$ and it was $w_{0}=35 \pm 1 \mu \mathrm{m}$. OA measurements were performed for different orientations of the linear polarization of the laser beam with respect to the plane of incidence (horizontal). The polarization was rotated by means of a half-wave plate.

\subsection{FEM simulations}

Simulations were carried out using the Finite Elements Method (FEM). ${ }^{34}$ The software COMSOL Multiphysics 5.1 was used to solve the Helmholtz equation in the frequency domain. Fig. 1c shows the 3D geometry of the model. In the horizontal $(\widehat{x y})$ plane, the array of nanoprisms was modeled by considering a hexagonal unit cell. In this configuration, the nanoprisms are placed at the vertices of the cell and each cell embeds a third of a prism per vertex. The tips of the prisms are oriented along the boundaries of the unit cell. Such a choice of the unit cell was done since, as we checked, it does not introduce differences with respect to the standard rhomboidal unit cell, but assures at the same time a greater numerical stability. In the vertical direction (i.e., the light propagation direction), the nanoprism base is on the $z=0$ plane, surrounded by silica on top and bottom. Over and below the simulated domains, two additional domains assure the absorption of unphysical backscattered radiation from external boundaries by using Perfectly Matched Layers (PML). The boundary conditions in the $\hat{x}$ and $\hat{y}$ directions are periodic (PBC). The $\mathrm{Ag}$ dielectric function used in the simulations was experimentally obtained by ellipsometry measurements performed on continuous films evaporated under the same conditions as those used for the NPAs. A J. Woolham V-VASE spectroscopic ellipsometer was used for these measurements. The refractive index of the silica layer was set to $n=1.45$. The shape of the nanoprisms was modeled using the FE-SEM images of the samples; their height was determined by AFM measurements. The mesh of the model was carried out by carefully assuring that it had the same hexagonal symmetry of the unit cell, to prevent spurious angular dependencies due to a non-uniform meshing. In the model, the nanoprisms were illuminated by plane waves traveling in the $\hat{z}$ direction. The angle of polarization of the incident light $(\theta)$ was varied from $\theta=0^{\circ}$ to $\theta=180^{\circ}$, with respect to the $\hat{x}$ direction in the $\widehat{x y}$ plane (see Fig. 1).

\section{Results and discussion}

By nanosphere lithography, ordered arrays of triangular nanoprisms arranged in a honeycomb lattice can be synthesized.$^{30} \mathrm{As}$ an example, Fig. 1b shows a FE-SEM image of the silver nanoprism arrays (NPAs) investigated in the present work. The image was taken before the deposition of the silica top layer (step 3 in the synthesis process shown in Fig. 1a). The geometrical parameters of the nanoarrays were: lattice parameter $a_{0}=248 \pm$ $5 \mathrm{~nm}$, nanoprism side length $L=45 \pm 1 \mathrm{~nm}$ and nanoprism height $h=40 \pm 1 \mathrm{~nm}$. The optical absorbance spectrum of the samples, measured after the silica deposition under unpolarized light illumination, is reported in Fig. 2a. Two peaks are visible in the spectrum, which can be interpreted as the dipolar (at $\lambda_{\mathrm{D}}=520 \mathrm{~nm}$ ) and the quadrupolar (at $\lambda_{\mathrm{Q}}=392 \mathrm{~nm}$ ) surface plasmon resonances (SPRs) of the nanoprism array. ${ }^{26,35,36}$ The linear absorption properties of the investigated NPA were also characterized as a function of the incident polarization. To do this, optical absorbance spectra were taken, with a linearly polarized light source, for different polarization orientations. The measurements are reported in Fig. 2b; the spectra are vertically shifted by a constant amount to improve the readability of the graph. The linear optical response of the NPA was found to be insensitive to the orientation of the incident electric field and no major differences were observed in the spectra. Such a behavior was expected taking into account the high degree of symmetry of the plasmonic lattice (honeycomb) in the samples, in agreement with literature data on similar samples ${ }^{37}$ and it was also confirmed by FEM simulations: consistent with the experimental findings, indeed, we did not highlight any dependence on the polarization in the simulated absorbance spectra.

The nonlinear absorption properties of the synthesized samples were studied using the z-scan technique in the open aperture (OA) configuration. ${ }^{31}$ The basic idea of this technique is to measure the intensity, transmitted by the sample, of a laser
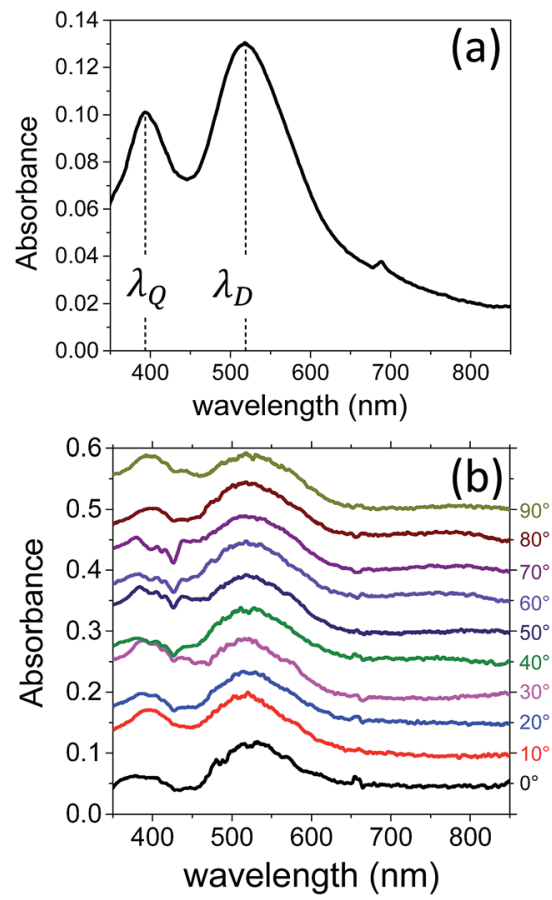

Fig. 2 (a) Optical absorbance spectrum of a Ag NPA taken with unpolarized light after the silica sputtering deposition (step 4 in Fig. 1a): the two peaks correspond to the dipolar (at $\lambda_{D}=520 \mathrm{~nm}$ ) and the quadrupolar (at $\lambda_{\mathrm{Q}}=392 \mathrm{~nm}$ ) surface plasmon resonances of the array. (b) Absorbance spectra as a function of the linear polarization orientation (in degrees, right-hand scale) of the incident light source; the spectra are vertically shifted by a constant amount to improve the readability of the graphs. 
beam focused by a converging lens, as a function of the position of the sample along the focal axis of the lens ( $z$ direction). In this way, the laser beam intensity on the sample varies with $z$, being maximum at the lens focus $(z=0)$, and nonlinear absorption processes can be revealed as changes in the measured transmittance. The intensity-dependent absorption coefficient is usually written in the form $\alpha(I)=\alpha_{0}+\beta I$, where $\alpha_{0}$ and $\beta$ are the linear and nonlinear absorption coefficients, respectively. The open aperture z-scan measurements allow both the magnitude and sign of the nonlinear absorption coefficient of the samples to be determined. Particularly, if saturable absorption (SA) phenomena are activated in the sample, they can be described by a negative nonlinear absorption coefficient $(\beta<0)$ and this would give rise to a peak in the OA transmittance scans at the lens focal point $(z=0)$. Conversely, reverse saturable absorption (RSA) processes are described by a positive nonlinear absorption coefficient $(\beta>0)$ and would be revealed by a minimum in the OA transmittance scans. In the present work, in order to reveal and investigate the possible effects of dichroism in the nonlinear absorption response of the samples, open aperture zscan measurements were performed for different orientations of the linear polarization of the incident laser beam. For this experiment, the second harmonic of a ps Nd:YAG laser $(\lambda=532$ $\mathrm{nm}$ ) was used to excite the dipolar resonance of the samples. All the measurements were performed with a laser peak intensity (i.e., the beam intensity at the focal point of the converging lens used in the z-scan set-up) of $I_{0}=280 \mathrm{MW} \mathrm{cm}^{-2}$. This prevented possible physical damage or modifications of the samples during the scans (as it was further checked by performing forward and backward scans). Fig. 3a shows the OA scan obtained with the incident polarization oriented at $0^{\circ}$ with respect to the plane of incidence (horizontal polarization). The continuous curve is the best fit to the experimental data obtained with the equation: ${ }^{31}$

$$
T_{\mathrm{OA}}=1-\frac{1}{2 \sqrt{2}} \frac{\beta I_{0} L_{\mathrm{eff}}}{\left(1+\left(z / z_{0}\right)^{2}\right)},
$$

where $z_{0}$ is the Rayleigh range $\left(z_{0}=\pi w_{0}{ }^{2} / \lambda\right)$, and $L_{\text {eff }}$ is the sample effective length $\left(L_{\text {eff }}=\frac{1-\mathrm{e}^{-\alpha_{0} L}}{\alpha_{0}}\right)$. The scan shows a maximum of transmittance at the lens focus $(z=0)$ that corresponds to a saturable absorption (SA) behavior. The response can be characterized in this case by a negative nonlinear absorption coefficient $(\beta<0)$. To perform OA measurements with different polarization orientations, the electric field of the incident beam was rotated by means of a half-wave plate with increments of $15^{\circ}$ with respect to the horizontal direction $\left(0^{\circ}\right)$, without changing the sample position. All the scans were fitted with eqn (1) and the results for the $\beta$ parameter are summarized in Fig. $3 \mathrm{~b}$ (red dots). A similar SA behavior was observed for all of the polarization orientations, ${ }^{38}$ but a continuous, sinusoidal modulation of the nonlinear absorption coefficient, $\beta$, was obtained as a function of the polarization angle. Such a modulation is well described by the function:

$$
\beta(\theta)=\beta^{*}+\Delta \beta \sin \left(\frac{2 \pi\left(\theta-\theta^{*}\right)}{T}\right)
$$

where $\beta^{*}$ is the average value of the nonlinear absorption coefficient $\left(\beta^{*}=\beta\left(\theta^{*}\right)\right), \Delta \beta$ is the amplitude of the modulation and $T$ is its period. By fitting the experimental data with eqn (2), we get the continuous curve in Fig. $3 \mathrm{~b}$, with the following set of parameters: $\beta^{*}=(-1.81 \pm 0.07) \times 10^{-4} \mathrm{~cm} \mathrm{~W}^{-1}, \Delta \beta=(0.51 \pm$ $0.09) \times 10^{-4} \mathrm{~cm} \mathrm{~W}^{-1}, \theta^{*}=(-9 \pm 4)^{\circ}$ and $T=(62 \pm 2)^{\circ}$. Thus, these findings indicate that, by working at $\lambda=532 \mathrm{~nm}$ (i.e., close to the dipolar resonance peak, $\lambda_{\mathrm{D}}=520 \mathrm{~nm}$ ), the investigated $\mathrm{Ag}$ nanoprism arrays exhibit a strong saturable absorber character $\left(\beta^{*}<0\right.$ and absolute values of the order of $\left.10^{-4} \mathrm{~cm} \mathrm{~W}^{-1}\right)$, and

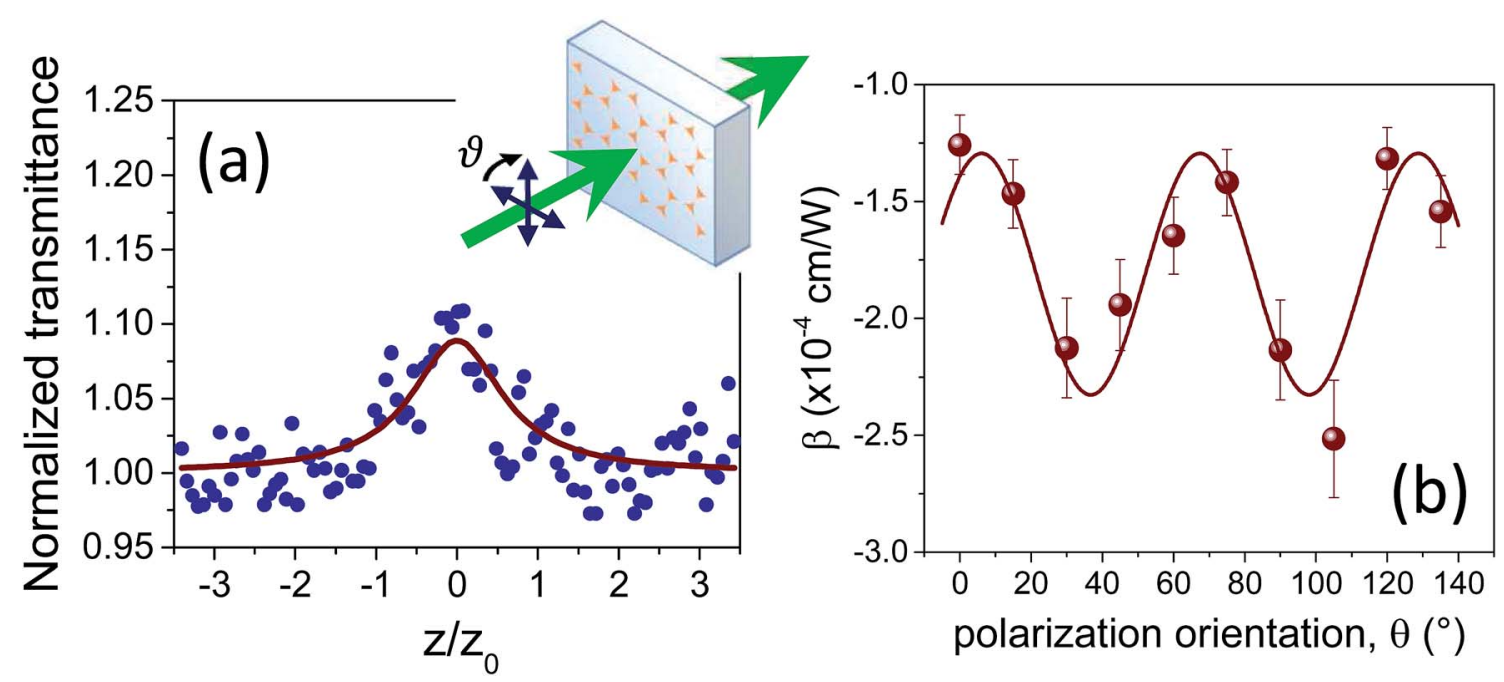

Fig. 3 (a) Open aperture $(O A) z$-scan of the Ag NPA measured with horizontal polarization of the laser beam $\left(\theta=0^{\circ}\right)$; the continuous curve is the best fit to the experimental data performed with eqn (1). The inset shows a sketch of the sample orientation with respect to the laser beam during the z-scan measurements. (b) Nonlinear absorption coefficient, $\beta$, as a function of the polarization orientation obtained by fitting the corresponding OA scans with eqn (1). All the measurements were taken at $\lambda=532 \mathrm{~nm}$, and the laser peak intensity was $I_{0}=280 \mathrm{MW} \mathrm{cm}^{-2}$. 
the magnitude of the SA response can be continuously modulated by rotating the incident linear polarization of the laser beam: $\Delta \beta / \beta^{*}=(29 \pm 5) \%$. Particularly, the estimated period of such a modulation is consistent with the $60^{\circ}$ symmetry of the NPA honeycomb lattice.

The effects of dichroism observed in the nonlinear absorption response of the investigated samples can be explained as a consequence of the local field enhancement in the NPA. Following the approach by Debrus et al. ${ }^{39,40}$ (recently used by Zheludev and co-workers to describe the nonlinear response of plasmonic metamaterials ${ }^{21}$ ), the nonlinear absorption coefficient of the NPA investigated in the present work can be expressed in terms of the distribution of local electric-field in the nanoprism array (E) according to the equation:

$$
\beta(\theta)=\operatorname{Re}\left\{\frac{\int \mathbf{E}^{2}(\theta)|\mathbf{E}(\theta)|^{2} \mathrm{~d} V}{\mathbf{E}_{0}^{2}\left|\mathbf{E}_{0}\right|^{2} V}\right\} \frac{n_{0}{ }^{2}}{n_{\mathrm{eff}}{ }^{2}} \beta_{\mathrm{m}}=\xi(\theta) \frac{n_{0}{ }^{2}}{n_{\mathrm{eff}}{ }^{2}} \beta_{\mathrm{m}}
$$

where the integral extends over the metal fraction in the unit cell volume $V$ (i.e., the volume of a composite layer of silica embedding the nanoprism array and with the same thickness as the nanoprism height). $E_{0}$ is the incident field amplitude. $n_{0}$ and $n_{\text {eff }}$ are the refractive index of bulk silver and the effective refractive index of the NPA, respectively, and $\beta_{\mathrm{m}}$ is the intrinsic nonlinear absorption coefficient of a homogeneous silver film. It was also assumed that the complex cubic susceptibility of the metal component is dominated by its imaginary part and that the nonlinear contribution of the silica matrix is negligible with respect to the metal one.

Therefore, according to eqn (3), an angle-dependent nonlinear absorption coefficient can be predicted for the investigated NPA due to the polarization-dependent local electric-field distribution in the array (i.e., the term $\xi(\theta)$ ). No angular dependence is instead expected for the other parameters in eqn (3). Particularly, the effective linear refractive index of the array, $n_{\text {eff }}$, was computed from the same field distribution data using the S-matrix method: ${ }^{41}$ the same value $\left(n_{\text {eff }}=0.198\right)$ was obtained for all the polarization orientations. In Fig. $4 \mathrm{a}-\mathrm{f}$ we report the electric field enhancement maps (at the $z=$ 0 plane), calculated using FEM simulations for the incident polarization oriented at the different indicated angles with respect to the $\hat{x}$ axis. The simulations were performed at the wavelength of the dipolar resonance peak $\left(\lambda_{\mathrm{D}}=520 \mathrm{~nm}\right)$. The maps show that the highest values of the electric-field enhancement factor, $F=|\mathbf{E}| / E_{0}$, are localized on the
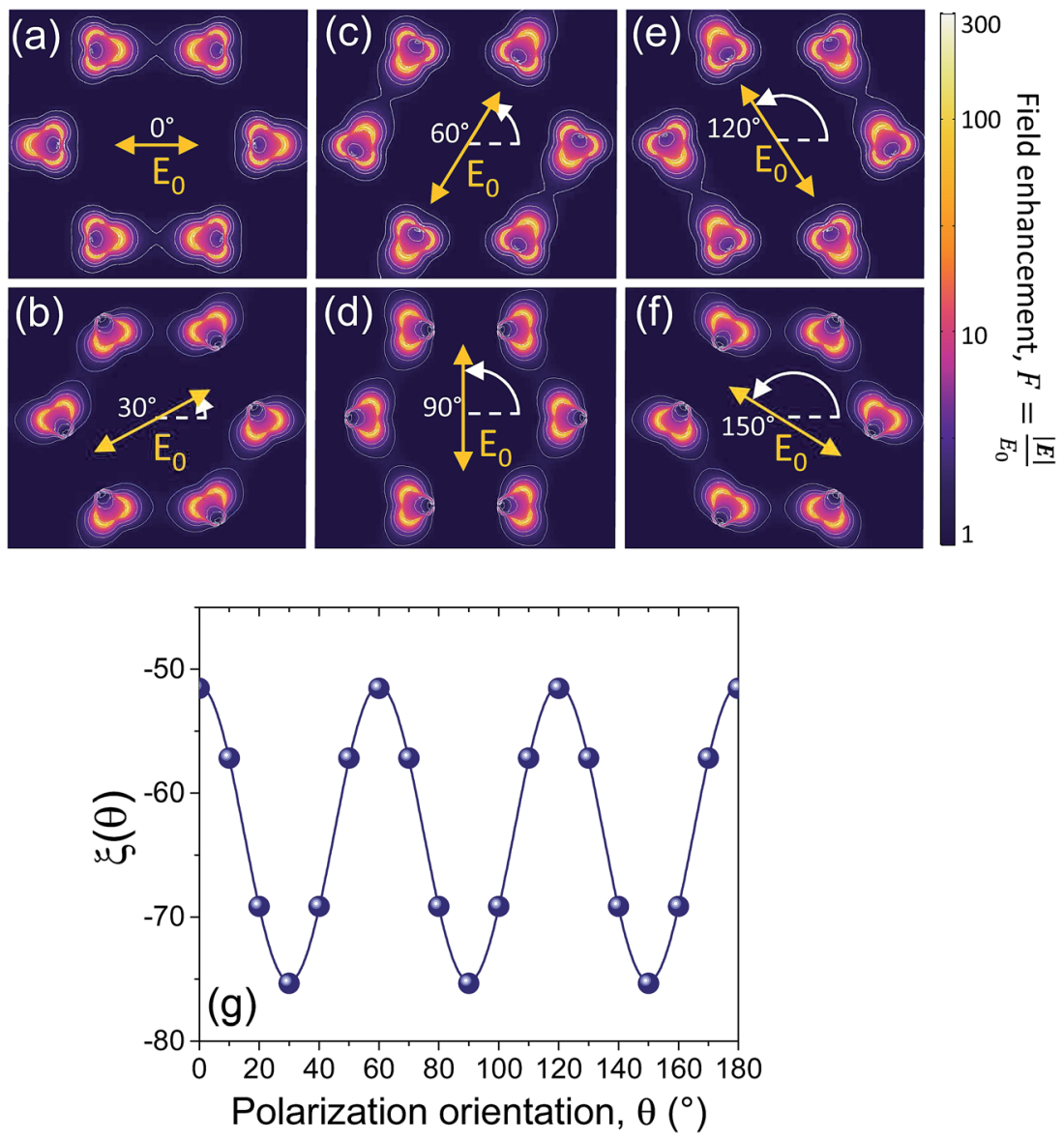

Fig. 4 (a-f) Simulated maps of electric field enhancement at the $z=0$ plane (silica substrate interface) evaluated at $\lambda_{\mathrm{D}}=520 \mathrm{~nm}$. The incident light beam is orthogonal to the sample surface and the electric field is linearly polarized at the different indicated angles with respect to the $\hat{x}$ direction (see Fig. 1c). The white contours indicate enhancement factors $F=1.5,3,5$ and 25. (g) Values of $\xi(\theta)$ in eqn (3) as a function of the polarization angle (blue dots); the blue curve is the best fit to the data performed with the sin function. 
nanoprism tips that are oriented along the direction parallel to the incident polarization. The white contours indicate the levels of enhancement factor $F=1.5,3,5$ and 25. These maps were used to compute the integral term $\xi(\theta)$ in eqn (3). The results of this calculation are reported in Fig. $4 \mathrm{~g}$ (blue dots). The data show an oscillating trend as a function of the polarization angle well described by the sin function (blue continuous curve) with a period of $60^{\circ}$, in perfect agreement with the experimental results shown in Fig. 3b. A modulation amplitude of about $20 \%$ of the average value is obtained in this case, still in agreement with the experimental result. A small phase shift is present between the simulated and the experimental data, due to the fact that, while for the simulations the polarization angle is perfectly defined with respect to the $\hat{x}$ direction in the unit cell of the array, an a priori alignment of the NPA with respect to the polarization direction of the laser beam during the z-scan measurements was not possible. Such a shift is taken into account in the parameter $\theta^{*}$ in eqn (2).

These findings further confirm the key role of the hot-spot formation in controlling the nonlinear optical properties of the investigated nanoprism arrays, giving rise to the demonstrated dichroic nonlinear absorption response. On the other hand, it is important to point out that if we calculate the average of the field enhancement factor over the whole unit cell of the array, no angular dependence comes out and a constant value $F=1.2$ is obtained for all the polarization orientations. This is due to the fact that the tip region is small compared to the unit cell (a volume ratio of about $4.3 \%$ was estimated), and thus the field enhancement averaged over the whole cell is weakly influenced by the hot-spots at the nanoprism tips. As a consequence, this explains why, differently from the nonlinear absorption response, the linear optical absorbance, which is dependent on the linear component of the electric field, does not exhibit any dependence on the incident polarization (Fig. 2).

\section{Conclusions}

The properties of dichroism in the nonlinear absorption response of $\mathrm{Ag}$ nanoprism arrays synthesized via nanosphere lithography were experimentally investigated using the z-scan technique. A picosecond laser beam with a low repetition rate was used to highlight the third-order electronic contribution in the optical nonlinearity of the samples, minimizing possible thermal effects. The measurements were performed by exciting the dipolar surface plasmon resonance of the synthesized nanoprism arrays. Open aperture z-scan measurements were performed for different linear polarization directions of the incident laser beam, in order to investigate the polarizationdependent nonlinear absorption properties of the investigated samples. The results showed that the samples have a strong saturable absorber character, and a continuous, sinusoidal modulation of the nonlinear absorption coefficient up to about $30 \%$ of its average value can be obtained by rotating the incident polarization. From FEM simulations, it was demonstrated that the observed dichroic nonlinear absorption behavior can be quantitatively explained by taking into account the polarization-dependent local electric-field distribution in the nanoarray. The properties highlighted in the present work make plasmonic nanoprism arrays a very interesting platform for the development of all-optical nanophotonic devices with fast, strong and tunable nonlinear optical response. As fast saturable absorbers, they could be used, for example, to develop solidstate mode-locking systems in ultra-fast laser devices, with low saturation intensity and in which the incident polarization can be exploited for the fine-tuning of the nonlinear absorption properties. Finally, as a last comment it has to be pointed out that the approach developed in the present work to explain the polarization-dependent nonlinear optical properties of the investigated nanoprism arrays can be used as well to characterize other plasmonic metamaterials. Thus, the incident polarization becomes an important parameter to take into account when comparing the nonlinear optical properties of different nanosystems.

\section{Acknowledgements}

This work has been supported by the Project of Major Importance Italy-México (Progetto di Grande Rilevanza Italia-Messico) MX14MO09 of the Italian Ministry of Foreigner Affairs and International Cooperation (MAECI). The authors thank CONACYT-México for grant 222485, and H. S. E. also thanks CONACYT for scholarship No. 221750 and mixed-funds grant 290842-359816. Financial support from PAPIIT-UNAM (México) through grant IN117116 is also acknowledged.

\section{References}

1 R. W. Boyd, Nonlinear Optics, Academic Press, 3rd edn, 2008.

2 X. Hu, Y. Zhang, Y. Fu, H. Yang and Q. Gong, Adv. Mater., 2011, 23, 4295-4300.

3 M. Kauranen, Science, 2013, 342, 1182-1183.

4 D. Cotter, R. J. Manning, K. J. Blow, A. D. Ellis, A. E. Kelly, D. Nesset, I. D. Phillips, A. J. Poustie and D. C. Rogers, Science, 1999, 286, 1523-1528.

5 R. A. Ganeev, Nonlinear Optical Properties of Materials, Springer, 2013.

6 M. Kauranen and A. V. Zayats, Nat. Photonics, 2012, 6, 737748.

7 S. A. Maier, Plasmonics: Fundamentals and Applications, Springer, New York, USA, 2007.

8 T. Cesca, P. Calvelli, G. Battaglin, P. Mazzoldi and G. Mattei, Opt. Express, 2012, 20, 4537-4547.

9 S. Gwo, C.-Y. Wang, H.-Y. Chen, M.-H. Lin, L. Sun, X. Li, W.-L. Chen, Y.-M. Chang and H. Ahn, ACS Photonics, 2016, 3, 1371-1384.

10 H. Harutyunyan, G. Volpe, R. Quidant and L. Novotny, Phys. Rev. Lett., 2012, 108, 217403.

11 P. Genevet, J. Tetienne, E. Gatzogiannis, R. Blanchard, M. A. Kats, M. O. Scully and F. Capasso, Nano Lett., 2010, 10, 4880-4883.

12 S. Kim, J. Jin, Y. Kim, I. Park, Y. Kim and S. Kim, Nature, 2008, 453, 757-760.

13 Y. Zhu, X. Hu, Y. Fu, H. Yang and Q. Gong, Sci. Rep., 2013, 3, 2338. 
14 R. Philip, P. Chantharasupawong, H. Qian, R. Jin and J. Thomas, Nano Lett., 2012, 12, 4661-4667.

15 A. L. Stepanov, Rev. Adv. Mater. Sci., 2011, 27, 115-145.

16 J. A. Reyes-Esqueda, V. Rodríguez-Iglesias, H.-G. SilvaPereyra, C. Torres-Torres, A.-L. Santiago-Ramírez, J. C. Cheang-Wong, A. Crespo-Sosa, L. RodríguezFernández, A. Lopez-Suarez and A. Oliver, Opt. Express, 2009, 17, 12849-12868.

17 A. López-Suárez, C. Torres-Torres, B. Can-Uc, R. Rangel-Rojo, C. E. Valencia and A. Oliver, J. Opt. Soc. Am. B, 2015, 32, 805811.

18 X.-L. Liu, J.-H. Wang, S. Liang, D.-J. Yang, F. Nan, S.-J. Ding, L. Zhou, Z.-H. Hao and Q.-Q. Wang, J. Phys. Chem. C, 2014, 118, 9659-9664.

19 G. A. Wurtz, R. Pollard, W. Hendren, G. P. Wiederrecht, D. J. Gosztola, V. A. Podolskiy and A. V. Zayats, Nat. Nanotechnol., 2011, 6, 107-111.

20 S.-W. Chu, H.-Y. Wu, Y.-T. Huang, T.-Y. Su, H. Lee, Y. Yonemaru, M. Yamanaka, R. Oketani, S. Kawata, S. Shoji and K. Fujita, ACS Photonics, 2014, 1, 32-37.

21 M. Ren, B. Jia, J.-Y. Ou, E. Plum, J. Zhang, K. F. MacDonald, A. E. Nikolaenko, J. Xu, M. Gu and N. I. Zheludev, Adv. Mater., 2011, 23, 5540-5544.

22 J. Y. Suh, M. D. Huntington, C. H. Kim, W. Zhou, M. R. Wasielewski and T. W. Odom, Nano Lett., 2012, 12, 269-274.

23 Y. Zhang, J. J. Wang, K. E. Ballantine, P. R. Eastham and W. J. Blau, Adv. Opt. Mater., 2014, 2, 331-337.

24 J. Butet and O. J. F. Martin, ACS Nano, 2014, 8, 4931-4939.

25 A. D. Neira, N. Olivier, M. E. Nasir, W. Dickson, G. A. Wurtz and A. V. Zayats, Nat. Commun., 2015, 6, 7757.

26 T. Cesca, N. Michieli, B. Kalinic, A. Sánchez-Espinoza, M. Rattin, V. Russo, V. Mattarello, C. Scian, P. Mazzoldi and G. Mattei, Nanoscale, 2015, 7, 12411-12418.
27 N. Michieli, PhD thesis, Doctoral School in Physics, 2014.

28 Z. Li, Y. Yu, Z. Chen, T. Liu, Z.-K. Zhou, J.-B. Han, J. Li, C. Jin and X. Wang, J. Phys. Chem. C, 2013, 117, 20127-20132.

29 K. O’Brien, H. Suchowski, J. Rho, A. Salandrino, B. Kante, X. Yin and X. Zhang, Nat. Mater., 2015, 14, 379-383.

30 J. C. Hulteen and R. P. Van Duyne, J. Vac. Sci. Technol., A, 1995, 13, 1553-1558.

31 M. Sheik-Bahae, A. Said, T. Wei, D. Hagan and E. V. Stryland, IEEE J. Quantum Electron., 1990, 26, 760-769.

32 G. Perotto, V. Bello, T. Cesca, G. Mattei, P. Mazzoldi, G. Pellegrini and C. Scian, Nucl. Instrum. Methods Phys. Res., Sect. B, 2010, 268, 3211-3214.

33 J. M. Khosrofian and B. A. Garetz, Appl. Opt., 1983, 22, 34063410 .

34 N. Michieli, B. Kalinic, C. Scian, T. Cesca and G. Mattei, Biosens. Bioelectron., 2015, 65, 346-353.

35 L. J. Sherry, R. Jin, C. A. Mirkin, G. C. Schatz and R. P. Van Duyne, Nano Lett., 2006, 6, 2060-2065.

36 N. Félidj, J. Grand, G. Laurent, J. Aubard, G. LÃlvivi, A. Hohenau, N. Galler, F. R. Aussenegg and J. R. Krenn, J. Chem. Phys., 2008, 128, 094702.

37 A. D. Humphrey and W. L. Barnes, Phys. Rev. B: Condens. Matter Mater. Phys., 2014, 90, 075404.

38 T. Cesca, H. Sanchez-Esquivel, E. Gemo, N. Michieli, B. Kalinic, E. V. García Ramírez, J. M. Gómez Cervantes, R. Rangel-Rojo, J. A. Reyes Esqueda and G. Mattei, Proc. SPIE 9894, Nonlinear Optics and its Applications IV, 2016, p. 98941A.

39 S. Debrus, J. Lafait, M. May, N. Pincon, D. Prot, C. Sella and J. Venturini, J. Appl. Phys., 2000, 88, 4469-4475.

40 H. Ma, R. Xiao and P. Sheng, J. Opt. Soc. Am. B, 1998, 15, 1022-1029.

41 D. Smith, D. Vier, T. Koschny and C. Soukoulis, Phys. Rev. E: Stat., Nonlinear, Soft Matter Phys., 2005, 71, 036617. 